\title{
Ground-based walking training improves quality of life and exercise capacity in COPD
}

Authors:

SL Wootton ${ }^{1,2}$, LWC Ng ${ }^{3,4}$, ZJ McKeough ${ }^{1}$, S Jenkins ${ }^{4,6,7}$, K Hill ${ }^{4,7}$, PR Eastwood ${ }^{4,5,11}$, DR Hillman $^{5}$, N Cecins ${ }^{6,7,10}$, LM Spencer ${ }^{8}$, C Jenkins ${ }^{9}$, JA Alison ${ }^{1,8}$

\section{Affiliations:}

(1) Clinical and Rehabilitation Sciences, The University of Sydney, New South Wales, Australia, 2006

(2) Chronic Disease Community Rehabilitation Service, Northern Sydney Local Health District, New South Wales, Australia, 2113

(3) Physiotherapy Department, Singapore General Hospital, Singapore, 169608

(4) School of Physiotherapy and Exercise Science, Curtin University, Western Australia, Australia, 6845

(5) Department of Pulmonary Physiology \& Sleep Medicine, Sir Charles Gairdner Hospital, Western Australia, Australia, 6009

(6) Physiotherapy Department, Sir Charles Gairdner Hospital, Western Australia, Australia, 6009

(7) Lung Institute of Western Australia and Centre for Asthma, Allergy and Respiratory Research, University of Western Australia, Western Australia, Australia, 6009

(8) Physiotherapy Department, Royal Prince Alfred Hospital, New South Wales, Australia, 2050

(9) Department of Thoracic Medicine, Concord Hospital, New South Wales, Australia, 2139

(10) Community Physiotherapy Services, Perth, Western Australia, Australia, 6000

(11) Centre for Sleep Science, School of Anatomy, Physiology \& Human Biology, University of Western Australia, Australia, 6009.

\section{Correspondence:}

Prof Jennifer Alison

75 East St Lidcombe

NSW Australia 2141 
Phone: +61293519371

Fax: +61293519278

E-mail: Jennifer.alison@sydney.edu.au

\section{Disclosures:}

This study was supported by an Australian NHMRC project grant: 570814

\section{Take-Home Message}

Walking training improves quality of life and endurance exercise capacity compared to usual medical care in COPD. 


\begin{abstract}
:
This study was designed to determine the effect of ground-based walking training on health-related quality of life and exercise capacity in people with COPD. People with COPD were randomised to either a Walk Group that received supervised, ground-based walking training, two to three times a week for eight to 10 weeks, or a Control Group that received usual medical care and did not participate in exercise training.

One hundred and thirty of 143 participants (mean [SD] age 69 [8] years [forced expiratory volume in one second $\left(\mathrm{FEV}_{1}\right) 43[15] \%$ predicted) completed the study. Compared to the Control Group, the Walk Group demonstrated greater improvements in the St George's Respiratory Questionnaire total score (mean difference -6 points, $95 \%$ CI -10 to $-2, p<0.003$ ), Chronic Respiratory Disease Questionnaire total score (mean difference 7 points, $95 \%$ CI 2 to $11, \mathrm{p}<0.01$ ) and endurance shuttle walk test time (mean difference 208 seconds, $95 \%$ CI 104 to 313 , $\mathrm{p}<0.001$ ). This study shows that ground-based walking training is an effective training modality that improves quality of life and endurance exercise capacity in people with COPD. (180 words)
\end{abstract}

\title{
Keywords:
}

Chronic obstructive pulmonary disease, pulmonary rehabilitation, exercise capacity, health-related quality of life, exercise test 


\section{INTRODUCTION}

Pulmonary rehabilitation has been shown to improve health-related quality of life (HRQoL) [1] and exercise capacity as well as reduce hospital admissions and length of stay in people with chronic obstructive pulmonary disease(COPD )[2-4]. Globally, the demand for pulmonary rehabilitation far outweighs the availability of programs [5-10]. Exercise training is considered to be the most important component of pulmonary rehabilitation [1] with endurance training most commonly undertaken on a treadmill or stationary cycle ergometer [1].

A simpler form of training requiring no equipment is ground-based walking. Although guidelines recommend that walking training can be incorporated within pulmonary rehabilitation programs [1], there is limited scientific evidence to support the use of ground-based walking training as the sole endurance training mode. Previous studies that have evaluated supervised, ground-based walking training have been limited by low participant numbers [11] and the lack of a control group that received no exercise intervention [12]. Establishing the effectiveness of ground-based walking as the sole exercise modality to improve exercise capacity and HRQoL, would increase the accessibility of exercise training for people with $\mathrm{COPD}$, especially in rural and remote locations where access to exercise equipment is limited.

The primary aim of this study was to determine the effects of a short-term, supervised, ground-based walking training program on HRQoL compared to usual medical care in people with COPD. A secondary aim was to determine the effects of ground-based walking training on endurance, functional and peak exercise capacity compared to usual medical care. We hypothesised that a supervised, ground-based walking training program would result in significant improvements in HRQoL and exercise capacity compared with usual medical care in people with COPD.

\section{METHODS}

\section{Participants}


Participants were recruited from referrals to outpatient pulmonary rehabilitation programs in two cities within Australia (Sydney, New South Wales and Perth, Western Australia). Participants were included in the study if they had a medical diagnosis of moderate, severe or very severe COPD according to the GOLD spirometric classification [13] (forced expiratory volume in one second $\left[\mathrm{FEV}_{1}\right]$ / forced vital capacity $\left.\mathrm{FVC}\right]$ of $<0.7$ and $\mathrm{FEV}_{1}<80 \%$ predicted) [14], were in a stable clinical state, and had a smoking history of greater than 10 pack years. Exclusion criteria were prescription of long term oxygen therapy, morbid obesity (body mass index $>35 \mathrm{~kg} / \mathrm{m}^{2}$ ), use of a walking aid, comorbidities likely to adversely affect exercise performance, or participation in supervised exercise training in the last 12 months. Written, informed consent was obtained from all participants.

\section{Study Design}

This study was a prospective, blinded (assessor and statistician), multi-centred, randomised controlled trial (RCT) with concealed allocation. Following baseline assessment participants were randomised via an independent telephone randomisation service using computerised random number generator sequencing, to a Walk Group or a Control Group. Randomisation was stratified according to baseline lung function $\left(\mathrm{FEV}_{1}<\right.$ or $\geq 40 \%$ predicted), HRQoL (St George's Respiratory Questionnaire [SGRQ] total score $<$ or $\geq 45$ ), exercise capacity (six-minute walk distance $[6 \mathrm{MWD}]<$ or $\geq 70 \%$ predicted) and study centre. Randomisation was biased towards the Walk Group with a 2:1 ratio for the purposes of a long-term follow-on study to 12 months. The study was approved by the ethics committees of Sydney South West Area Health Service, The University of Sydney, Curtin University, Sir Charles Gairdner Hospital and Bentley Hospital. The trial was registered in the Australia and New Zealand Clinical Trials Registry (ACTRN12609000472279).

\section{Intervention}

Participants in the Walk Group performed ground-based walking training while participants in the Control Group did not participate in any exercise training and were not given any instructions regarding exercise. A letter was sent to the general medical practitioner of each participant to advise 
them of their patient's involvement in the study and to encourage optimal medical management. The letter included the Australian COPD best practice management guidelines [15] and a COPD action plan that recommended individualised medication use when stable and during an exacerbation [15].

\section{Walk Training}

Participants in the Walk Group attended walking training sessions three times a week for eight weeks. Walking training was performed on a flat indoor track within the participating hospitals and was supervised by physiotherapists experienced in providing pulmonary rehabilitation for people with COPD. The ground-based walking training commenced at 30 minutes duration with the speed set at $80 \%$ of the average speed achieved during the six-minute walk test (6MWT) [16]. Ground-based walking training was progressed by five minutes after every $6^{\text {th }}$ training session to a maximum of 45 minutes. Participants were instructed to walk at a pace which elicited a dyspnoea score of 3-4 on a modified 0-10 point category-ratio dyspnoea scale [17]. Walking speed (intensity) was progressed on an individual basis depending on each participant's ability. If speed could not be increased due to a limitation in stride length and the participant was scoring $<3$ on the dyspnoea scale, weight belts were worn during training. The starting weight was $2.5 \mathrm{~kg}$ and was increased by $1 \mathrm{~kg}$ after every third session if the participant reported a dyspnoea score $<3$ at the end of the training session.

Immediately prior to, and at the end of, all supervised walking training sessions, heart rate (HR) and oxygen saturation $\left(\mathrm{SpO}_{2}\right)$ were recorded using a finger probe connected to either a Masimo Rad 5 (Masimo Corporation, Irvine, CA, USA) or a Novametric (Respironics, Murrysville, PA, USA) oximeter. Rest breaks were allowed during training in the event of intolerable symptoms, and HR and $\mathrm{SpO}_{2}$ were assessed during rest breaks using a pulse oximeter. Participants were encouraged to recommence walking training as soon as they felt able and rest time was not included in the duration of the training session. 
If a participant was unable to attend three times per week, he/she was required to attend twice a week and complete one unsupervised session of walking training per week or attend twice a week for 10 weeks. The aim was to ensure that at least 20 walking training sessions were completed within eight to ten weeks. In the event of a participant having an exacerbation of COPD or being unable to attend due to illness, the exercise training was extended by a maximum of two weeks. Training compliance was measured by the number of completed training sessions.

\section{Lung Function Tests}

Spirometry $\left(\mathrm{FEV}_{1}\right.$ and $\left.\mathrm{FVC}\right)$ was completed at baseline and on study completion using a calibrated portable spirometer (EasyOne spirometer, ndd Medical Technologies Inc., Andover, MA, USA) according to standard procedures [18]. Lung volumes (body plethysmography) and single breath diffusion capacity of the lung $\left(\mathrm{D}_{\mathrm{L}}, \mathrm{CO}\right)$ were measured at baseline only, according to standard protocols [19, 20]. Measures obtained were compared to normative data [14, 21, 22]. Disease severity of each participant was classified according to the GOLD spirometric criteria [13].

\section{Outcome Measures}

All outcomes were measured at baseline and at study completion. The primary outcomes were HRQoL measured by the SGRQ and the Chronic Respiratory Disease Questionnaire (CRQ). Secondary outcomes were endurance exercise capacity measured by the endurance shuttle walk test (ESWT) time, functional exercise capacity measured by the 6MWT and peak exercise capacity measured by the incremental shuttle walk test (ISWT).

\section{Measurement of HRQoL}

HRQoL was measured with the SGRQ [23] and the interviewer-administered CRQ with an individualised dyspnoea domain [24]. The SGRQ has 53 items examining the impact of respiratory disease across three domains (symptoms, activity limitations and impact of disease) of HRQoL. The CRQ is a COPD specific measurement tool that has 20 questions examining four domains of HRQoL 
(dyspnoea, fatigue, emotional function and disease mastery). During follow up interviews, respondents were reminded of their responses to the most recently completed CRQ and asked if they felt the same, better or worse.

\section{Measurement of Exercise Capacity}

Participants completed two ESWTs, two 6MWTs and two ISWTs over two visits within a seven day period. On the first visit, both ISWTs were completed prior to one ESWT. During the second visit the second ESWT was performed followed by two 6MWTs. The ISWT and 6MWT were performed according to standardised protocols $[25,26]$. Since the ESWT has a ceiling effect (maximum 20 minute test duration), the protocol [27] was modified so that if a participant completed more than 10 minutes in the first ESWT, the test was terminated and repeated at the higher level so that the participant's baseline ESWT time was between five and 10 minutes. This was done to allow the opportunity for improvement at the reassessment and minimise the ceiling effect. . The 6MWT track varied according to site and was either rectangular (26 to 32 metres) or straight (20 to 45 metres). At each site, participants performed all 6MWTs on the same track. Repeat tests performed on the same day were separated by a 30 minute rest period. The test that yielded the greatest distance was recorded as the test outcome. The distances achieved on the ISWT and 6MWT were compared with normative data $[28,29]$. During all walk tests, $\mathrm{SpO}_{2}$ (Pulse oximeter; Masimo Rad 5, Masimo Corporation, Irvine, CA, USA or Novametric, Respironics, Murrysville, PA, USA) and HR (Polar heart rate monitor; (Polar Electro, Finland) were continuously monitored. If $\mathrm{SpO}_{2}$ fell below $80 \%$ during testing, the ISWT was terminated and, in the 6MWT, a rest was imposed until $\mathrm{SpO}_{2}$ increased to $\geq$ 90\%. Dyspnoea and rate of perceived exertion (RPE) were assessed before and at the end of each exercise test using the modified 0-10 point category-ratio scale [17]. During the ESWT, dyspnoea and RPE were assessed at one minute intervals in order to compare ratings at isotime. Isotime was defined as the end time of the pre- or post-intervention ESWT, whichever was the shorter.

\section{Sample Size}


The sample size was based on detection of a meaningful difference in the mean total CRQ score between the Walk Group and the Control Group. The recommended minimum important difference for the total CRQ score is 10 points [30]. Allowing for a $20 \%$ loss to follow up and assuming a standard deviation (SD) of 17 points for total CRQ score [31], 132 participants were sufficient to provide $80 \%$ power with an alpha of 0.05 (two-sided) (after adjusting for group size imbalance). This sample size was also sufficient to detect a -4 point difference in SGRQ total score, which is the minimum important difference for SGRQ [32] .

\section{Data Analysis}

Data are presented as mean and SD unless otherwise stated. Data were analysed using SPSS software (Version 20 for Windows, SPSS Inc, Chicago, ILL, USA). A p-value $\leq 0.05$ was considered significant. Analysis of covariance was used with baseline values as the covariate to assess differences between groups for all data. Intention to treat analysis was conducted with no imputation of missing values. Independent samples t-tests were used to assess the differences between groups in the isotime data.

\section{RESULTS}

\section{Participant Characteristics}

Participant flow is presented in Figure 1. One hundred and thirty of 143 participants, completed the study (mean (SD) age 69 (8) years, $\mathrm{FEV}_{1} 43$ (15) \% predicted). Baseline characteristics of participants were similar between groups (Table 1). Participants had moderate to severe COPD [13], reduced HRQoL (mean (SD) SGRQ total score 47 (17)) and reduced exercise capacity ((mean (SD) 6MWD $74 \%$ (13) predicted). Thirteen (9\%) participants in the Walk Group did not complete the postintervention outcome measures for the following reasons: four (3\%) withdrew due to medical conditions other than COPD; three (2\%) were admitted to hospital (two with a COPD exacerbation and one with a bowel obstruction); three (2\%) were unable to be contacted; two (1\%) discontinued the intervention and declined reassessment; and one (1\%) declined to attend for re-assessment. Of these 
13 participants, five did not commence any training sessions. There were no differences in baseline lung function ( $\mathrm{FEV}_{1} \%$ predicted, $\left.\mathrm{p}=0.53\right)$, HRQoL (SGRQ total score, $\mathrm{p}=0.97$ ) or exercise capacity (6MWD \% predicted, $\mathrm{p}=0.18$ ) between the participants who completed or did not complete the study. The modification to the ESWT protocol resulted in the ESWT level (speed) being increased for 36 (25\%) participants at the baseline assessment.

In the Walk Group, $75 \%$ of sessions (i.e. 18 (6) out of a possible 24 sessions) were completed by the 90 participants who commenced walking training. Fifty-three participants $(56 \%)$ rested during training due to dyspnoea or fatigue. Eleven (9\%) of the 90 participants used weight belts during training. The walking distances achieved by the Walk Group during training showed a progressive increase (Figure 2), with 76 (84\%) participants progressing their walking duration. Of those who progressed, $89 \%$ were in GOLD II, $85 \%$ were in GOLD III and 67\% were in GOLD IV. Dyspnoea ratings remained constant between 'moderate' to 'somewhat severe' as the training duration increased (Figure 2). No adverse events were reported with the walking training.

\section{HRQoL}

Within group differences in HRQoL showed significant improvement in total scores and all domains of both the SGRQ and the CRQ in the Walk Group but not the Control Group (Table 2). Compared to the Control Group, the Walk Group demonstrated greater improvements in the SGRQ total score and the SGRQ domains of activity limitations and impact of disease, as well as CRQ total score and the CRQ domains of emotional function and disease mastery (Table 2).

\section{Exercise Capacity}

Within group analyses showed there was a significant increase in ISWT distance and ESWT time in the Walk Group (Table 2), but not the Control Group. Compared to the Control Group, the Walk Group demonstrated greater improvements in ESWT time and 6MWD, although the latter was 
partially due to a non-significant decrease in walk distance in the Control Group (Table 2). There were no differences between groups in ISWT distance (Table 2). At study completion, 15 (18\%) participants in the Walk Group and two (4\%) participants in the Control Group completed 20 minutes on the ESWT. When measurements were compared at isotime during the ESWT (Table 3) a small but significant decrease in dyspnoea was noted in the Walk Group, however, there were no differences in dyspnoea or RPE between the Walk Group and Control Group at isotime (Table 3).

\section{DISCUSSION}

Many pulmonary rehabilitation programs are designed around cycling and treadmill walking being the primary training modalities [1]. There has been a growing interest in the effectiveness of groundbased walking programs to improve HRQoL and exercise capacity, given that ground-based walking training is simple to perform, readily available and easy to administer as it requires no exercise equipment. This is the first large randomised controlled trial of the effects of supervised ground-based walking training on HRQoL and exercise capacity compared with a control group receiving usual care and no exercise intervention, in people with moderate to severe COPD. The outcome measures used in this study were comprehensive and included two disease specific questionnaires to evaluate HRQoL and three different types of walking exercise tests to evaluate exercise capacity. The main findings of this study were that ground-based walking training improved HRQoL and endurance walking capacity when compared to usual care.

We consider it a notable finding that walking training significantly improved HRQoL, especially given that no other mode of training or any disease management education was provided. The few studies that have examined the effect of ground-based walk training on HRQoL have also shown improvements $[12,33,34]$. These programs have varied in the delivery of walking training being either supervised [12], unsupervised [33] or involving Nordic walking [34]. All studies had relatively small sample sizes (20 to 30 participants per group) thereby limiting the ability to both generalise the findings and determine a precise estimate of the magnitude of the effect that walking training had on 
HRQoL. Our study, in a much larger cohort, confirms these early findings that ground-based walking training can improve HRQoL. The key components of walking training which we believe were important to achieve improved HRQoL were the individualised walking intensity based on the initial 6MWT and supervision by physiotherapists experienced in pulmonary rehabilitation enabling continued monitoring to ensure appropriate progression of intensity and duration of walking training.

Two HRQoL questionnaires were used as primary outcome measures in this study. The total score of the SGRQ showed a significant and clinically relevant improvement (a reduction in score by $\geq 4$ points) [23] in the Walk Group compared to the Control Group with significant improvements in the subscores of 'Activity Limitation' and 'Impacts' between groups. For the CRQ total score, the between group difference did show a significant improvement, but this did not reach the minimum important difference for this outcome [30]. The types of questions within each HRQoL questionnaire may explain the difference in responsiveness of the two HRQoL measures. Many of the activity questions within the SGRQ were focused on activities involving walking whereas for the CRQ, participants chose activities that caused them to be short of breath and these activities may not have involved walking and, therefore, may not have been altered by the effects of the intervention.

The secondary aim of the study was to determine the effects of supervised, ground-based walking training on endurance, functional and peak exercise capacity. Ground-based walking training improved endurance exercise capacity, measured by the ESWT, in the Walk Group compared to the Control Group. The improvement in ESWT time was 71\% greater in the Walk Group compared to the Control Group. This improvement exceeded the distribution-based minimum important difference for pulmonary rehabilitation of 186 seconds [35]. It is likely that the observed improvement in endurance exercise capacity was an underestimation of the true increase since 15 participants in the Walk Group completed 20 minutes on the ESWT at the final assessment, which reduced the responsiveness of this measure to detect change. In terms of functional exercise capacity, measured by the 6MWT, there was a small, statistically significant between group difference in 6MWD of $22 \mathrm{~m}$ but this was largely a 
consequence of a small non-significant reduction in 6MWD in the Control Group rather than an improvement in the Walk Group. This small improvement did not reach the reported minimum important difference following pulmonary rehabilitation of 25 metres [36]. In terms of peak exercise capacity, while the Walk Group showed a small statistically significant improvement in ISWT distance of 25 metres, this improvement did not result in a statistically significant or clinically important between group difference.

In order to improve 6MWD and ISWT distance, participants must be able to walk faster. While walking training was effective at improving endurance exercise capacity, it did not improve the average speed walked over six minutes in the 6MWT or the peak walking speed in the ISWT, suggesting that walking training enabled participants to walk further but not faster. Given that the program of walking training focussed on increasing walking duration rather than increasing walking speed it is possible that participants were conditioned to walk at a particular speed during the posttraining tests. Importantly, improvement in walking endurance rather than speed may make a greater difference to a person's ability to perform activities of daily living. In the present study, the improvement in the 'Activity Limitation' subscale of the SGRQ would support this proposition.

There were no adverse events reported during walking training despite the relatively long walking durations of up to 45 minutes. During the training sessions, dyspnoea levels remained stable while duration of training sessions and distances achieved increased (Figure 2). Such a finding indicated that the participants were able to train for progressively longer durations without increased levels of dyspnoea, reflecting the improvement in exercise tolerance achieved at study completion. Given the ease of transferability of walk training, this mode of training could be transposed to other settings (e.g. community). However, the key principles applied in this study would need to be adhered to, namely, individually determined training prescription from baseline exercise testing and adequate supervision of training to enable appropriate training progression. Walking training may also be easily 
implemented in the home environment, enabling participants to continue this mode of exercise in the longer term with potential maintenance of benefits.

The study had a low drop-out rate of $9 \%$ which is in contrast with higher attrition from clinical pulmonary rehabilitation programs [2]. One reason for this difference may be that 'drop out' from a clinical pulmonary rehabilitation program is often reported when patients do not attend training, whereas, in a clinical trial, participants remain in the trial as long as some of the final assessments are performed (i.e intention-to-treat). Interestingly, there were no drop-outs in the Control Group, most likely because this group had no exercise intervention and the only requirement was to perform final assessment testing. Following participation in the study the Control Group participants were offered standard pulmonary rehabilitation which may have been an incentive to attend for re-assessment.

A limitation of the study was that detailed physiological testing was not performed. This limited our ability to explain the mechanisms of improvement in endurance exercise capacity in the Walk Group compared to the Control Group. One indication of a training effect was the small but significant reduction in dyspnoea at isotime during the ESWT suggesting that ventilatory demand may have been reduced at an equivalent work rate after training in the Walk Group. However, such a reduction in dyspnoea could also have been due to reduced levels of anxiety or improved neuromuscular recruitment. More detailed studies would be required to elucidate the physiological effects of walking training. A further study limitation was that people with mild COPD (GOLD I) were not recruited, therefore we cannot readily extend the study findings to this group.

\section{Conclusion}

In conclusion, ground-based walking training improved HRQoL and endurance exercise capacity compared to usual medical care in people with COPD. The robust design of this study provides strong support for ground-based walking training having a therapeutic role as an endurance training 
modality for people with moderate to severe COPD, and this may be particularly applicable where specialised exercise equipment is not readily available.

(3648 words)

Acknowledgements: The authors would like to thank the staff at Royal Prince Alfred Hospital, Concord Repatriation General Hospital, Prince of Wales Hospital, Manly Hospital, Hornsby Ku-ringgai Hospital, Bentley Hospital and Sir Charles Gairdner Hospital for their assistance and support, thank the patients who participated in the study and thank Professor Jennifer Peat for statistical support.

Competing interests: None

Funding: Australian National Health and Medical Research Council project grant: 570814. PR Eastwood is funded by a National Health and Medical Research Council of Australia (NHMRC) Senior Research Fellowship (1042341). 


\section{REFERENCES:}

1. Spruit MA, Singh SJ, Garvey C, et al. An Official American Thoracic Society / European Respiratory Society Statement: Key concepts and advances in pulmonary rehabilitation - An Executive Summary. Am J Respir Crit Care Med 2013;188(8):e13-e64.

2. Cecins N, Geelhoed E, Jenkins S. Reduction in hospitalisations following pulmonary rehabilitation in patient with COPD. Australian Health Review. 2008;32(3):415-22.

3. Griffiths T, Phillips C, Davies S, et al. Cost effectiveness of an outpatient mulitdisciplinary pulmonary rehabiliation programme. Thorax. 2001;56:779-84.

4. Rubí MD, Renom F, Ramis F, et al. Effectiveness of pulmonary rehabilitation in reducing health resources use in chronic obstructive pulmonary disease. Arch Phys Med Rehabil 2010;91(3):364-8.

5. ALF. Case statement: chronic obstructive pulmonary disease. . Australian Lung Foundation http://wwwlungnetcomau 2001.

6. Kida K, Jinno S, Nomura K, et al. Pulmonary rehabilitation program survey in North America, Europe, and Tokyo. J Cardiopulm Rehabil. 1998 Jul-Aug;18(4):301-8. PubMed PMID: 9702610. Epub 1998/08/14. eng.

7. Yohannes AM, Connolly MJ. Pulmonary rehabilitation programmes in the UK: a national representative survey. Clin Rehabil. 2004;18(4):444-9.

8. Levack WMM, Weatherall M, Reeve JC, et al. Uptake of pulmonary rehabilitation in New Zealand by people with chronic obstructive pulmonary disease in 2009. N Z Med J. 2012;125 (1348):23-33.

9. Brooks D, Sottana R, Bell B, et al. Characterization of pulmonary rehabilitation programs in Canada 2005. Canadian Respiratory Journal. 2007;14(2):87-92.

10. Wadell K, Janaudis Ferreira T, Arne M, et al. Hospital-based pulmonary rehabilitation in patients with COPD in Sweden - A national survey. Respir Med. 2013;107:1195-200.

11. Lake FR, Henderson K, Briffa T, et a. Upper-limb and lower-limb exercise training in patients with chronic airflow obstruction. Chest. 1990;97:1077-82.

12. Leung RWM, Alison JA, McKeough ZJ, Peters MJ. Ground walk training improves functional exercise capacity more than cycle training in people with chronic obstructive pulmonary disease (COPD): a randomised trial. Journal of Physiotherapy. 2010;56:105-12.

13. Global Initiative for Chronic Obstructive Lung Disease. Global strategy for the diagnosis, management, and prevention of COPD [04/07/2012]. Available from: www.goldcopd.org

14. Hankinson J, Odencrantz J, Fedan K. Spirometric reference values from a sample of the general US population. Am J Respir Crit Care Med. 1999;159:179-87.

15. McKenzie DK, Abramson MJ, Crockett AJ, Glasgow N, Jenkins S, McDonald C, et al. The COPD-X Plan: Australian and New Zealand Guidelines for the management of Chronic Obstructive Pulmonary Disease. 2007.

16. Jenkins S, Hill K, Cecins NM. State of the art: How to set up a pulmonary rehabilitation program. Respirology. 2010;15:1157-73.

17. Borg G. Psychophysical bases of perceived exertion. Med Sci Sports Exer. 1982;14:377-81.

18. Miller MR, Hankinson J, Brusasco V, et al. Standardisation of spirometry. Eur Respir J.

2005;26:319-38.

19. Wanger J, Clausen J, Coates A, et al. Standardisation of the measurement of lung volumes. Eur Respir J. 2005;26(511-22).

20. MacIntyre N, Crapo RO, Viegi G, et al. Standardisation of the single-breath determination of carbon monoxide uptake in the lung. Eur Respir J. 2005;26:720-35.

21. Stocks J, Quanjer P. Reference values for residual volume, functional residual capacity and total lung capacity. Eur Respir J. 1995;8:492-506.

22. Crapo RO, Morris AH. Standardised single breath normal values for carbon monoxide diffusing capacity. Am Rev Resp Dis. 1981;123:185-9.

23. Jones PW, Quirk FH, Baveystock CM. The St George's Respiratory Questionnaire (SGRQ). Respir Med 1991;85:25-31. 
24. Guyatt GH, Berman LB, Townsend M, et al. A measure of quality of life for clinical trials in chronic lung disease. Thorax. 1987;42:773-8.

25. Singh S, Morgan M, Scott S, et al. Development of a shuttle walking test of disability in patients with chronic airways obstruction. Thorax. 1992;47:1019-24.

26. American Thoracic Society. Guidelines for the six-minute walk test. Am J Respir Crit Care Med 2002; 166:111-117. 2002; 166:111-7.

27. Revill S, Morgan M, Singh S, et al. The endurance shuttle walk: A new field test for the assessment of endurance capacity in Chronic Obstructive Pulmonary Disease. Thorax. 1999;54(3):213-22.

28. Probst V, Hernandes N, Teixeira D, et al. Reference values for the incremental shuttle walking test. Respir Med. 2012;106:243-8.

29. Jenkins S, Cecins N, Camarri B, et al. Regression equations to predict six-minute walk distance in middle-aged and elderly adults. Physiother Theory Pract. 2009;25(7):516-22.

30. Jaeschke R, Singer J, Guyatt GH. Measurement of health status. Ascertaining the minimal clinically important difference. Controlled Clinical Trials. 1989;10:407-15.

31. Ries AL, Kaplan RM, Myers R, Prewitt LM. Maintenance after pulmonary rehabilitation in chronic lung disease. American Journal of Respiratory \& Critical Care Medicine. 2003;167:880-8.

32. Jones PW. Interpreting thresholds for a clinically significant change in health status in asthma and COPD. Eur Respir J. 2002;19(3):398-404.

33. Hernández MTE, Rubio TM, Ruiz FO, Et al. Results of a home-based training program for patients with COPD. Chest 2000;118:106-14.

34. Breyer MK, Breyer-Kohansal R, Funk GC, et al. Nordic Walking improves daily physical activities in COPD: a randomised controlled trial. Respir Res. 2010;11(112).

35. Pepin V, Laviolette L, Brouillard C, et al. Significance of changes in endurance shuttle walking performance. Thorax. 2011;66(2):115-20.

36. Holland AE, Hill CJ, Rasekaba T, et al. Updating the minimal important difference for sixminute walk distance in patients with chronic obstructive pulmonary disease. Arch Phys Med Rehabil. 2010;91:221-5. 
Table 1: Participant Characteristics

\begin{tabular}{lcc}
\hline Variable & Walk Group & Control Group \\
$\mathrm{n}=95$ & $68(9)$ \\
\hline Age, yr & $69(8)$ & $68(9)$ \\
Gender, male/female & $56 / 39$ & $1.68(0.11)$ \\
Height, $\mathrm{m}$ & $1.67(0.10)$ & $78(19)$ \\
Weight, kg & $71(15)$ & $27(6)$ \\
BMI, kg/m ${ }^{2}$ & $25(5)$ & 10 \\
Current smokers, $\mathrm{n}$ & 15 & \\
Pulmonary function & & $1.19(0.47)$ \\
FEV, L & $1.13(0.42)$ & $43(15)$ \\
FEV $\%$ predicted & $43(15)$ & $2.76(0.83)$ \\
FVC, L & $2.71(0.84)$ & $75(18)$ \\
FVC \% predicted & $76(17)$ & $0.43(0.12)$ \\
FEV $/$ FVC ratio & $0.43(0.14)$ & $109(18)$ \\
TLC \% predicted & $114(32)$ & $139(37)$ \\
FRC \% predicted & $153(60)$ & $147(51)$ \\
RV \% predicted & $162(82)$ & $0.52(0.11)$ \\
RV/TLC ratio & $0.54(0.09)$ & $43(15)$ \\
$D_{\mathrm{L}}, \mathrm{CO} \%$ predicted & $44(17)$ & $22(46 \%)$ \\
GOLD grade & & $21(44 \%)$ \\
II & $40(42 \%)$ & $5(10 \%)$ \\
III & $42(44 \%)$ & \\
IV & $13(14 \%)$ & \\
\hline
\end{tabular}

Data presented as mean (SD).yr: years m: metres; kg: kilograms; $\mathrm{kg} / \mathrm{m}^{2}$ : kilograms per metre squared; BMI: body mass index; L: litre; $\mathrm{FEV}_{1}$ : forced expiratory volume in 1second; FVC: forced vital capacity; TLC: total lung capacity; FRC: functional residual capacity; RV: residual volume; $\mathrm{D}_{\mathrm{L}}, \mathrm{CO}$ : single breath diffusing capacity for carbon monoxide. 
Table 2: Walk test and questionnaire data in the Walk Group (WG) and Control Group (CG) participants at baseline and study completion

\begin{tabular}{|c|c|c|c|c|c|c|c|c|}
\hline \multirow[t]{3}{*}{ Outcome } & \multicolumn{2}{|c|}{ Baseline } & \multicolumn{2}{|c|}{ Study completion } & \multicolumn{2}{|c|}{ Within group } & \multirow{3}{*}{$\begin{array}{l}\text { Between group } \\
\text { Mean difference } \\
\quad(95 \% \mathrm{CI}) \\
\end{array}$} & \multirow{3}{*}{$\begin{array}{c}\text { ANCOVA } \\
\text { p value }\end{array}$} \\
\hline & \multicolumn{2}{|c|}{ Mean (SD) } & \multicolumn{2}{|c|}{ Mean (SD) } & \multicolumn{2}{|c|}{$\begin{array}{l}\text { Mean difference } \\
\qquad(95 \% \mathrm{CI})\end{array}$} & & \\
\hline & WG & $\mathrm{CG}$ & WG & CG & WG & $\mathrm{CG}$ & & \\
\hline ISWT, metres & $319(115)$ & $332(118)$ & $344(132)$ & 335 (127) & $25(10,40)^{*}$ & $3(-13,18)$ & $22(-0.2,44)$ & 0.052 \\
\hline ESWT, seconds & $330(208)$ & $294(168)$ & $574(389)$ & $333(227)$ & $245(174,315)^{*}$ & $38(-29,106)$ & $208(104,313)^{\#}$ & $<0.001$ \\
\hline $6 \mathrm{MWT}$, metres & $465(85)$ & $473(91)$ & $474(92)$ & $459(90)$ & $9(-1,19)$ & $-14(-29,0.3)$ & $22(6,39)^{\#}$ & 0.01 \\
\hline \multicolumn{9}{|l|}{ SGRQ } \\
\hline Total score & $47(17)$ & $47(16)$ & $41(14)$ & $47(16)$ & $-6(-9,-3)^{*}$ & $0.1(-4,4)$ & $-6(-10,-2)^{\#}$ & 0.003 \\
\hline Symptoms & $56(22)$ & $62(21)$ & $50(23)$ & $59(21)$ & $-6(-10,-1)^{*}$ & $-3(-9,3)$ & $-5(-12,2)$ & 0.19 \\
\hline Activity limitations & $63(19)$ & $64(20)$ & $59(18)$ & $66(20)$ & $-5(-7,-2)^{*}$ & $2(-2,7)$ & $-7(-11,-2)^{\#}$ & 0.003 \\
\hline Impacts & $33(18)$ & $33(17)$ & $27(14)$ & $33(16)$ & $-6(-9,-3)^{*}$ & $-0.3(-5,4)$ & $-6(-10,-1)^{\#}$ & 0.01 \\
\hline \multicolumn{9}{|l|}{ CRQ } \\
\hline Total score & $89(19)$ & $89(17)$ & $97(18)$ & $90(18)$ & $8(5,11)^{*}$ & $1(-3,5)$ & $7(2,11)^{\#}$ & 0.01 \\
\hline Dyspnoea & $16(5)$ & $17(5)$ & $19(5)$ & $18(6)$ & $2(1,3)^{*}$ & $1(-0.4,2)$ & $1(-0.4,3)$ & 0.15 \\
\hline Fatigue & $17(6)$ & $17(4)$ & $19(5)$ & $17(4)$ & $1(0.4,2)^{*}$ & $1(-0.3,2)$ & $1(-0.4,2)$ & 0.18 \\
\hline Emotional Function & $35(8)$ & $34(9)$ & $37(7)$ & $35(9)$ & $3(1,4)^{*}$ & $0.2(-2,2)$ & $3(1,5)^{\#}$ & 0.01 \\
\hline Mastery & $21(5)$ & $21(5)$ & $22(5)$ & $21(5)$ & $1(1,2)^{*}$ & $0.2(-1,1)$ & $1(0.1,3)^{\#}$ & 0.04 \\
\hline
\end{tabular}

Data presented as mean (SD) or mean difference $(95 \% \mathrm{CI})$ between groups. WG: walk group; CG: control group; ISWT: incremental shuttle walk test (WG $\mathrm{n}=74$; $\mathrm{CG}$ n=47); ESWT: endurance shuttle walk test (WG $\mathrm{n}=78, \mathrm{CG} \mathrm{n}=47$ ); 6MWT: six-minute walk test (WG n=77, CG n= 45); SGRQ: St George's Respiratory Questionnaire (WG $\mathrm{n}=80$, CG n=45), CRQ: Chronic Respiratory Disease Questionnaire (WG n=81, CG n=48)

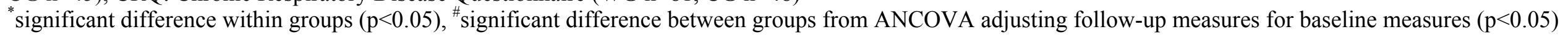


Table 3: Group data for dyspnoea and RPE at isotime during the ESWT

\begin{tabular}{|c|c|c|c|c|c|c|c|c|}
\hline & \multirow{2}{*}{\multicolumn{2}{|c|}{$\frac{\text { Baseline }}{\text { Mean (SD) }}$}} & \multirow{2}{*}{\multicolumn{2}{|c|}{$\begin{array}{c}\text { Study completion } \\
\text { Mean (SD) }\end{array}$}} & \multirow{2}{*}{\multicolumn{2}{|c|}{$\begin{array}{c}\text { Within group } \\
\text { Mean difference } \\
(95 \% \mathrm{CI})\end{array}$}} & \multirow{3}{*}{$\begin{array}{c}\text { Between group } \\
\text { Mean difference } \\
(95 \% \mathrm{CI})\end{array}$} & \multirow{3}{*}{$\frac{\text { ANCOVA }}{p \text { value }}$} \\
\hline & & & & & & & & \\
\hline & WG & CG & WG & CG & WG & CG & & \\
\hline \multirow[t]{2}{*}{ Dyspnoea, score } & 4.5 & 4.2 & 3.9 & 4.1 & $-0.6(-0.1,-1.0)^{*}$ & $-0.1(-0.6,0.4)$ & $0.5(-0.2,1.2)$ & 0.13 \\
\hline & $(2.0)$ & (1.7) & (1.8) & (1.7) & & & & \\
\hline \multirow[t]{2}{*}{ RPE, score } & 4.5 & 4.6 & 4.2 & 4.4 & $-0.4(-0.8,0.0)$ & $-0.2(-0.8,0.5)$ & $0.2(-0.48,0.95)$ & 0.52 \\
\hline & $(2.0)$ & $(2.0)$ & (1.7) & (1.7) & & & & \\
\hline
\end{tabular}

Data presented as mean (SD) of groups, mean (SD) difference within groups, and mean (95\% CI) difference between groups. Isotime was defined as the end time of the pre- or post-intervention ESWT, whichever was the shorter. WG: Walk Group; CG: Control Group; RPE: Rate of Perceived Exertion * significant difference within groups $(\mathrm{p}<0.05)$ 


\section{FIGURE LEGENDS}

Figure 1: Participant flow through the study. $W G=$ Walk Group; $C G=$ Control Group

Figure 2: Peak walking distances and dyspnoea ratings during the walking training program. Dashed line $=$ dyspnoea. Solid line $=$ distance walked in training. Error bars $=$ standard error, $\mathrm{m}=$ metres 
Figure 1 - Participant flow

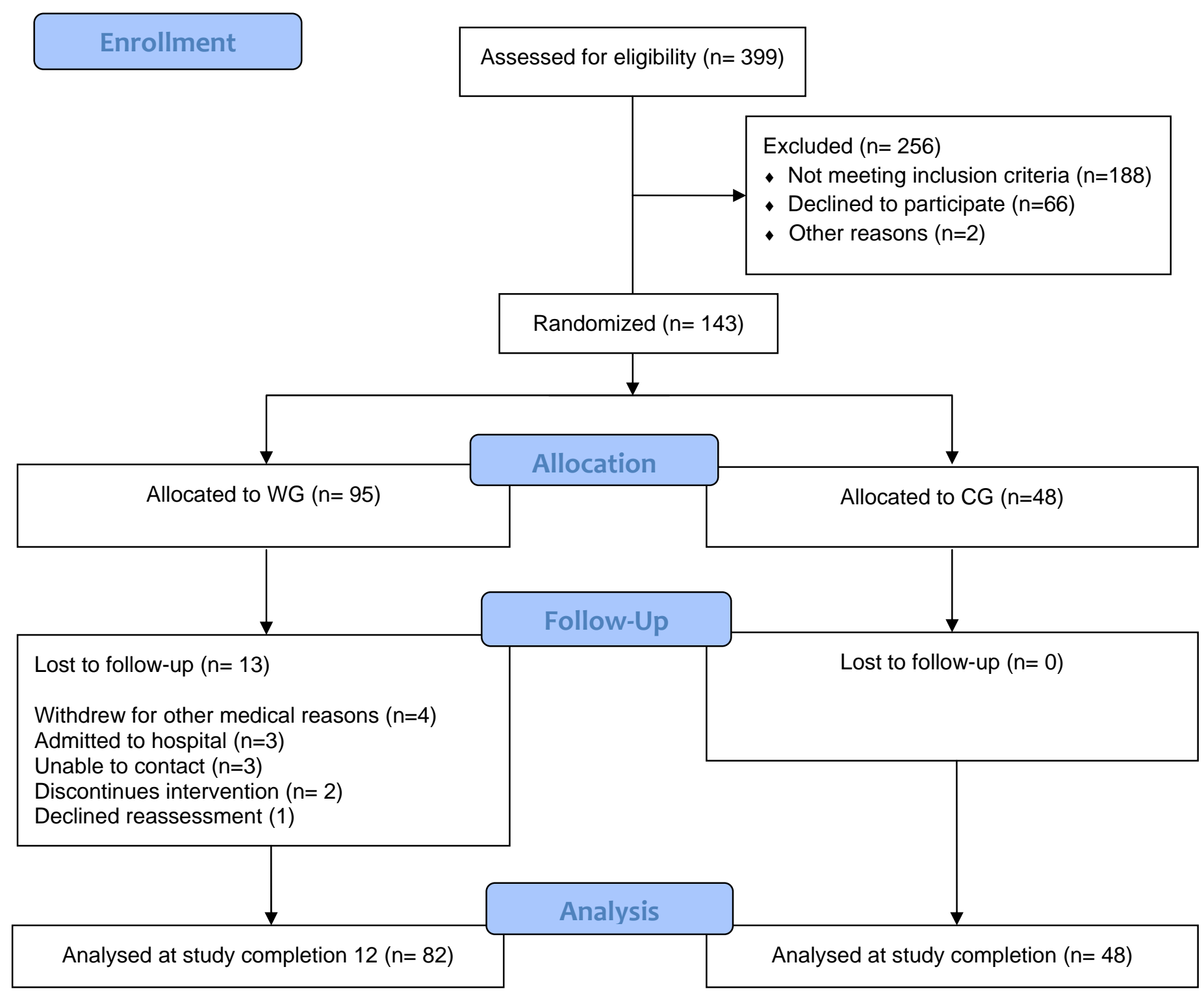


Figure 2

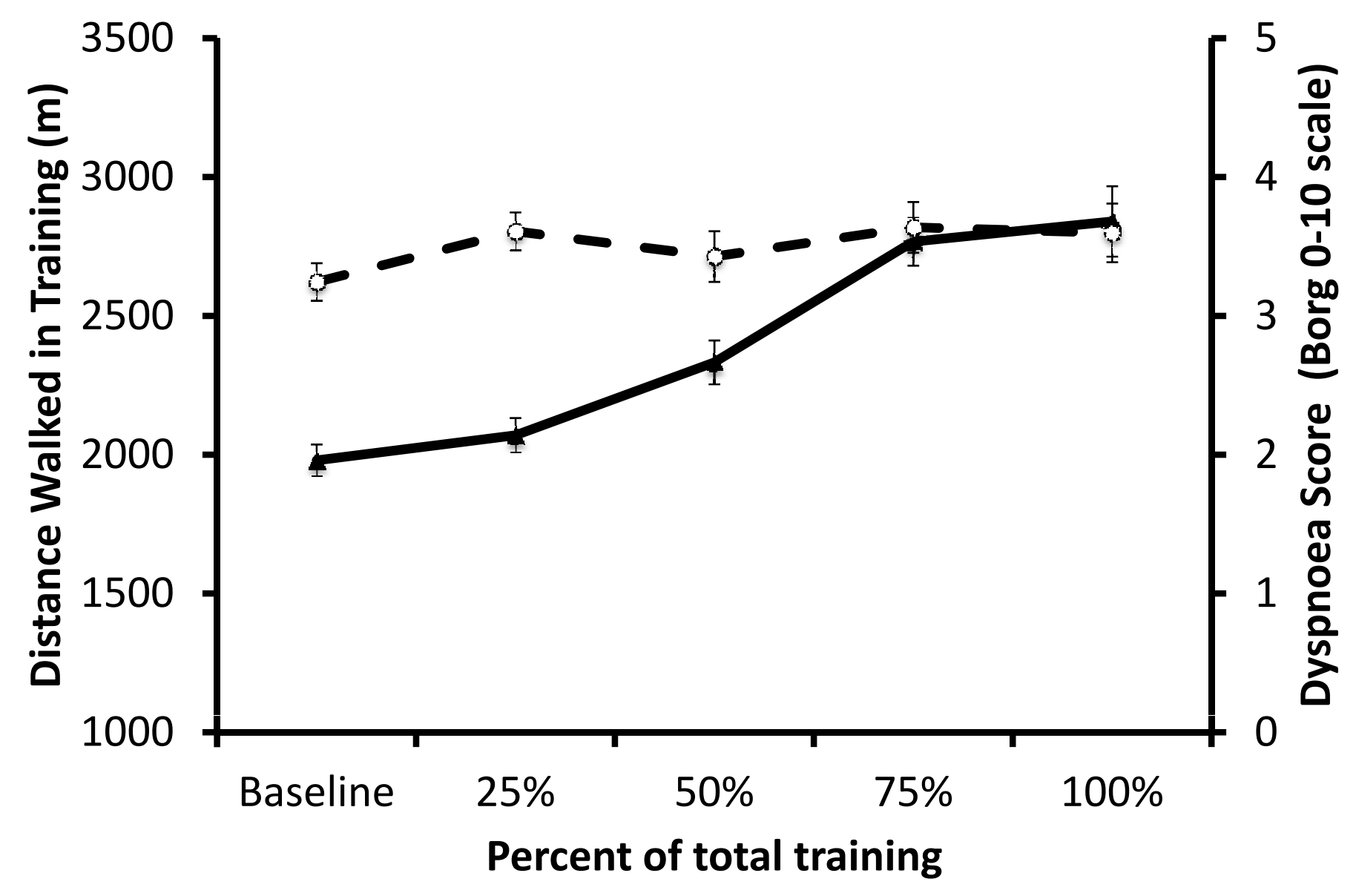

